\title{
System for 3D Visualization of Flaws for Eddy Current Inspection
}

\begin{abstract}
A. Dočekal, M. Kreidl, R. Šmíd
This paper presents a novel method for 3D visualization of flaws detected during Eddy Current (EC) inspection. The EC data was acquired using an automated scanning system equipped with precise eddy current probe positioning. The method was tested on a single frequency instrument with an absolute probe. The EC inspection procedure is implemented statically by registering the operating point of the instrument at each equidistant point on a tested object.

The paper describes a data processing method based on the Fourier transform enabling 3D visualization of flaws. This three-dimensional image of the result of a scan enables the position offlaws to be determined, and the size and bevel (angle to the surface) of each detected flaw to be estimated. This research investigated flaws rising from the surface of the tested object, and flaw depth was not evaluated in this work. This method of visualization is simple to implement and is currently targeted for application in EC scanning devices.
\end{abstract}

Keywords: Eddy Current (EC), visualization, modified Fourier descriptors.

\section{Introduction}

The Eddy Current (EC) method is a well-established technique for flaw detection and sizing in many application areas of non-destructive testing (NDT), e.g. in aircraft maintenance. A typical task is inspection for cracks close to the surface of tested material.

An automated scanning system using motorized eddy current (EC) probe positioning is often applied for testing objects with large surfaces. There are several important benefits of enabling users to see 3D images of a surface containing detected flaws (EC scan). In particular, a three-dimensional image of the result of a scan enables personnel to determine the approximate position of flaws and to estimate the size (length) and bevel of each detected flaw immediately (flaw depth is not evaluated here).

Eddy current phenomena can be modeled by nonlinear three-dimensional, partial differential equations with complicated boundary conditions. However, modeling-based analysis methods are difficult to apply for test data evaluation, so in automated analysis systems EC signatures, as a trajectory of the instrument produced signal in the complex plane, are usually considered [1]. The complex signal $s(n)$ consists of an in-phase (horizontal) component $h(n)$ and a quadrature (vertical) component $v(n)$.

Modeling of flaws and 3D visualization can be faithfully done by simulating the electromagnetic field with a 3D finite element method [2]. Since 3D finite element calculations are very time consuming and this kind of method requires high-powered equipment for to calculate and calibrate it, it may be interesting to introduce a less sophisticated but accurate method that requires fewer computer resources.

This paper is organized as shown in the block diagram of the EC scanning system, see Fig. 1. Section 2 describes the data acquisition stage and the appropriate equipment configuration. Section 3 focuses on the main data processing methods (pre-processing and feature extraction). Section 4 outlines the visualization that is used. Finally, Section 5 contains conclusions.

\section{Data acquisition}

The EC scanning system consists of two independent components. A single frequency instrument with an absolute probe forms the main component. An absolutely shielded EC probe with active diameter cca $2 \mathrm{~mm}$ was used. The probe was

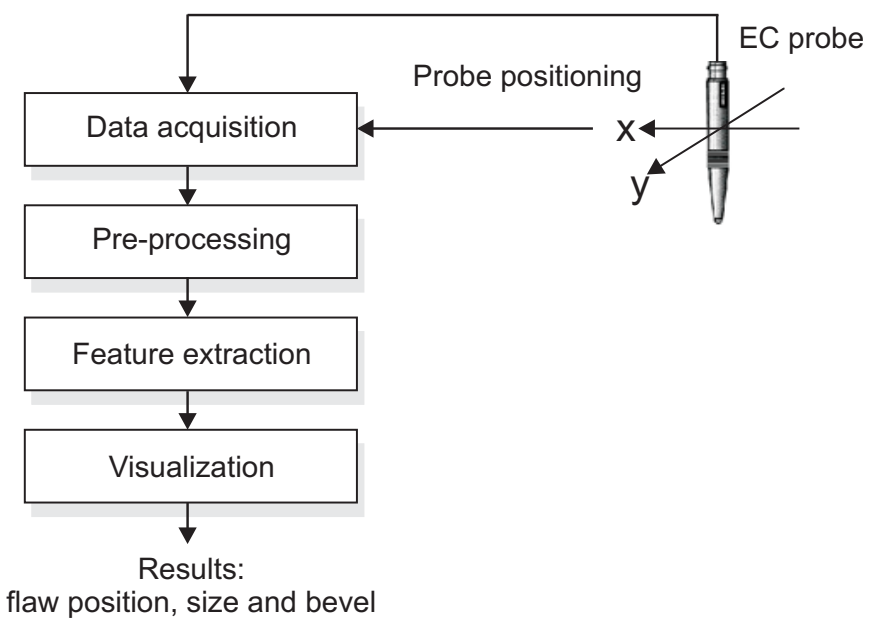

Fig. 1: Block diagram of an automated EC scanning system 


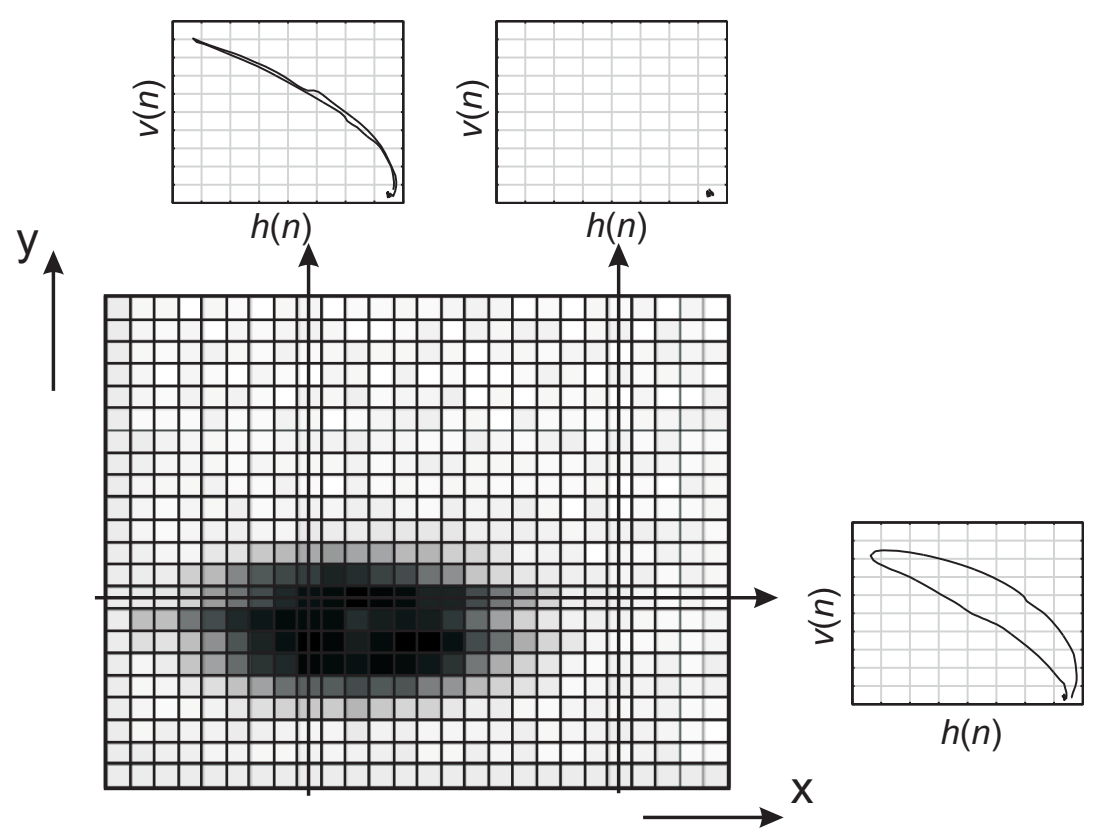

Fig. 2: Matrix representing data acquired during the test with the corresponding EC signatures

operated at a frequency of $200 \mathrm{kHz}$ to achieve a sufficient penetration depth and sufficient resolution. The other part was posed by a precise EC probe positioning system. The positioning system achieved resolution $0.125 \mathrm{~mm}$ in the probe movement. A probe shift step of $0.3 \mathrm{~mm}$ was used for material testing.

The two instruments did not enable on-the-fly scanning. Hence the scanning process had to be synchronized by data acquisition software and the scan procedure was implemented statically. The surface of the scanned object was tested at equidistant points in order to ensure data synchronization. The EC signal value was registered or more precisely the mean of the signal samples of the EC tester at each equidistant point. A matrix formed of EC signal values resulted from the data acquisition process, see Fig. 2. Each row and each column of this matrix represents one EC signature describing an EC inspection for an assigned direction and probe shift, as shown in Fig. 2.

\section{Signal processing}

The main aim of the visualization method is to provide personnel with information about the occurrence of a flaw on the surface, and to estimate the position, surface orientation, size (depth) and bevel (angle to surface) of each detected flaw.

Information about the position and surface orientation of a flaw can be obtained approximately by evaluating the threshold of the absolute value of the complex EC signal, or more precisely the absolute difference from the reference value. In this way, the orientation of the flaw surface convolved with the spatial response of the used EC probe is obtained. The position and surface orientation of a flaw can be given more accurately by applying an inverse filtering procedure, as described in [4]. To estimate the uncertainty carried in the simple signal thresholding, an EC probe response to a reference orthogonal flaw with sufficient surface orientation and size $1.5 \mathrm{~mm}$ on the tested material was measured, see Fig. 3.
In the measured case the uncertainty was estimated at $0.9 \mathrm{~mm}$ $(k=2)$.

The shape (size and bevel) of a flaw is determined from the shapes of the appropriate EC signatures of the flaw in each related row and column of the matrix. These EC signatures correspond to standard testing in the relevant direction of the probe movement (horizontal or vertical) and the probe offset to the initial position, shown in Fig. 2. The EC signature shape is described using the Fourier transform, described under Feature extraction, see Section 3.2.

The description of a flaw at each tested point calculated from the EC signatures can be represented as a $3 \mathrm{D}$ vector. The flaws are visualized as an approximation of the $3 \mathrm{D}$ vector endpoints by flaw surface forming.

\subsection{Pre-processing}

A number of pre-processing steps were carried out to reduce the impact of disturbances especially outliers caused by noise. The EC signature and the corresponding signal were filtered by a non-linear filtration method called Median signature tracking. This method is based on median filtering an EC signature in the complex plane. The curvature of the EC signature is tracked using the median computed from the angles of the corresponding samples $\boldsymbol{s}(n)$. The median signature tracking procedure has been described in [5].

\subsection{Feature extraction}

To extract shape-describing features from the EC signatures, we used a method based on the Fourier transform. Standard Fourier descriptors are based on expanding the signature shape in a Fourier series:

$\boldsymbol{F}(p)=\frac{1}{N} \sum_{n=0}^{N-1} \boldsymbol{s}(n) e^{-j \frac{2 \pi}{N} n p}$, for $p=0,1,2, \ldots,(N-1)$

where $\boldsymbol{s}(n)$ is an EC signature of length $N$. For feature extraction a limited number of coefficients are selected. We 


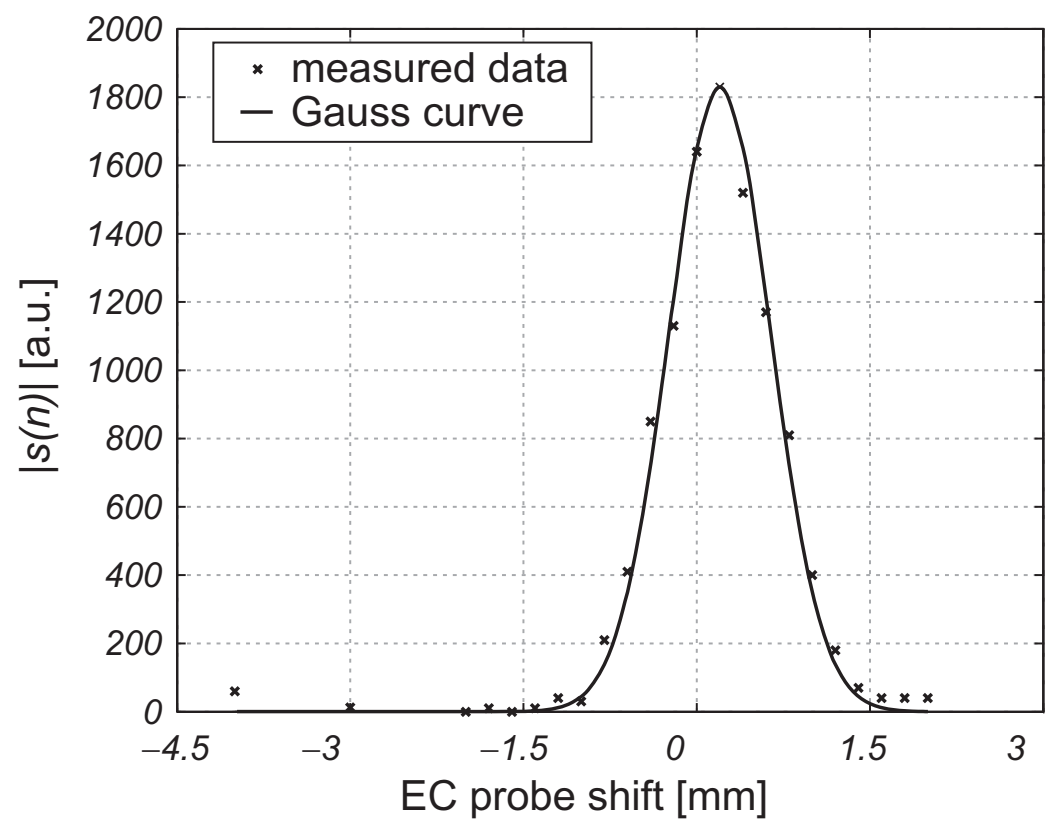

Fig. 3: Spatial response of the used EC probe to the reference flaw (a.u. arbitrary unit)

normalize the descriptors with respect to signature translations (especially offset) [5].

$f_{n 1}(r)=|\boldsymbol{F}(r)|+|\boldsymbol{F}(N-r)|$,

$f_{n 2}(r)=\frac{|\boldsymbol{F}(r)| \cdot|\boldsymbol{F}(N-r)|}{\max (|\boldsymbol{F}(r)|,|\boldsymbol{F}(N-r)|)^{2}}$,

$f_{n 3}(r)=\frac{\arg |\boldsymbol{F}(r)|+\arg |\boldsymbol{F}(N-r)|}{2}$,

where $r$ is descriptor order, $r \neq 0$. Modified Fourier descriptors (MFD) $f_{n 1}, f_{n 2}$ and $f_{n 3}$ correspond to overall size, ellipticity and angle of signature description by harmonic $r$ respectively. We can estimate the flaw size (length), bevel and depth (for flaws drowned in the material). The orientation of a flaw can be simply recognized from the direction of the EC value movement at an EC signature (complex curve) though the row or column of the matrix. Flaw depth was not assessed in our study, which deals only with flaws rising from surface of a tested object.

\subsection{Visualization}

The position and surface orientation of each flaw is taken by processing the absolute value of the EC signal, see Fig. 2 .

The first step in the visualization process is to assign a three-dimensional vector to the given features. These vectors are found for each point at which the flaw and the surface intersect. We used two EC signatures that intersected at a certain point on the surface, and these were measured for two orthogonal directions (horizontal and vertical) of the EC probe movement, see Fig. 2. This stage can be split into two parts.

First, we calculate a vector that represents the information found from the shape and size of one EC signature (in one direction). The calculation uses Modified Fourier descriptors, and only first order MFD descriptors were used.

Secondly, we construct the three-dimensional vector representing information about the flaw at the examined point in both orthogonal directions on the surface. This vector collects information from both EC signatures measured in orthogonal directions. The first vector, representing the horizontal part, gives values of $d_{f}$ and $h_{f}$, while the second vector, representing the vertical part, gives values of $d_{f}$ and $v_{f}$ (see Fig. 4.). The final $3 \mathrm{D}$ vector is given as the maximum of each component. Finally, a surface is formed from these vectors.

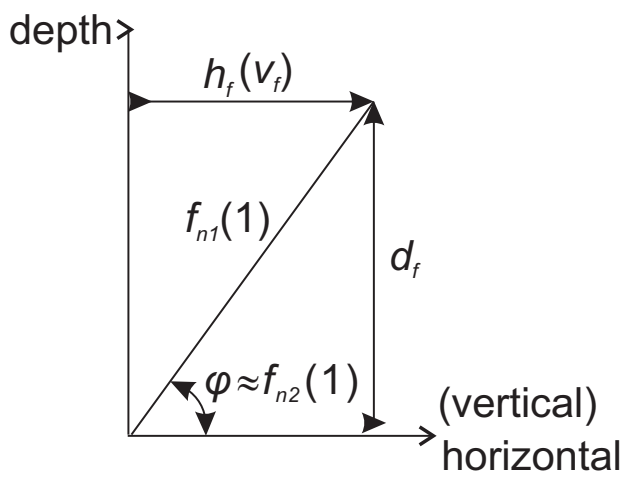

Fig. 4: Construction of a 3D representation of an EC signature

The two operations can be expressed by equations (5) to (8), illustrated in Fig. 4,

$$
\begin{aligned}
& f_{n 2}(1) \approx \sin \varphi, \\
& d_{f}=f_{n 1}(1) \cdot f_{n 2}(1),
\end{aligned}
$$

whereas equation (7) is used for assigning the vector representing the horizontal part, and the equation (8) assigns the vector representing the vertical part.

$h_{f}=f_{n 1}(1) \cdot \sqrt{1-f_{n 2}^{2}(1)}$ and $v_{f}=0$,

$v_{f}=f_{n 1}(1) \cdot \sqrt{1-f_{n 2}^{2}(1)}$ and $h_{f}=0$.

This result requires calibration especially due to the non-linear principle of EC inspection. This requires calibration 
measurements on a sample made of the tested material. This sample should include the possibility of calibrating each considered feature as size and bevel. Only after calibration can unknown samples be tested. This is a major disadvantage of the method. Polynomial curve fitting was applied to calibrate all considered features. We used a polynomial $p(x)$ of degree 2 that fits the data in a least squares sense (Eq. 9).

$$
p(x)=p_{2} x^{2}+p_{1} x+p_{0} .
$$

Calibration of the size descriptor $f_{n 1}(1)$ for an aluminium sample is illustrated in Fig. 5.

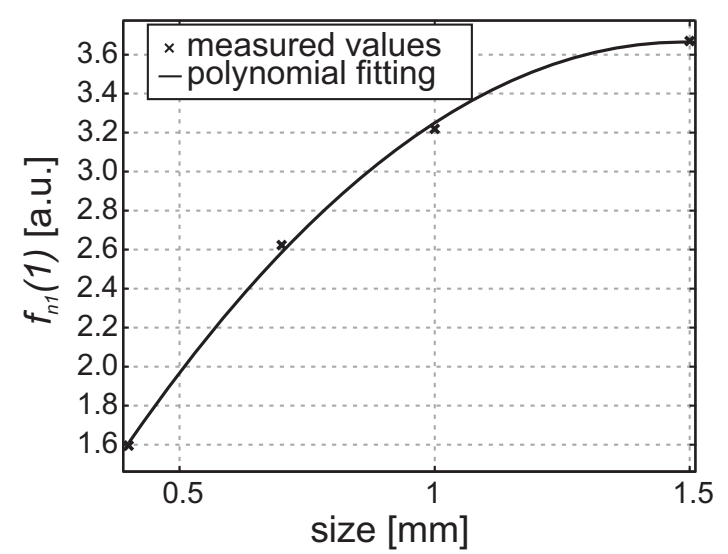

Fig. 5: Calibration data of the size acquired on the first sample

\section{Experimental evaluation}

This visualization method was evaluated in tests of two aluminium samples. The first sample contained notches of $0.3 \mathrm{~mm}$ width, size $0.4,0.7,1$ and $1.5 \mathrm{~mm}$ perpendicular, depth $0.4,0.7,1$ and $1.5 \mathrm{~mm}$ with an angle of $30^{\circ}, 0.7,1$ and $1.5 \mathrm{~mm}$ with an angle of $60^{\circ}$, and $1.5 \mathrm{~mm}$ with an angle of $45^{\circ}$. This sample was made up of the same material as the second sample, which was also used for test calibration.

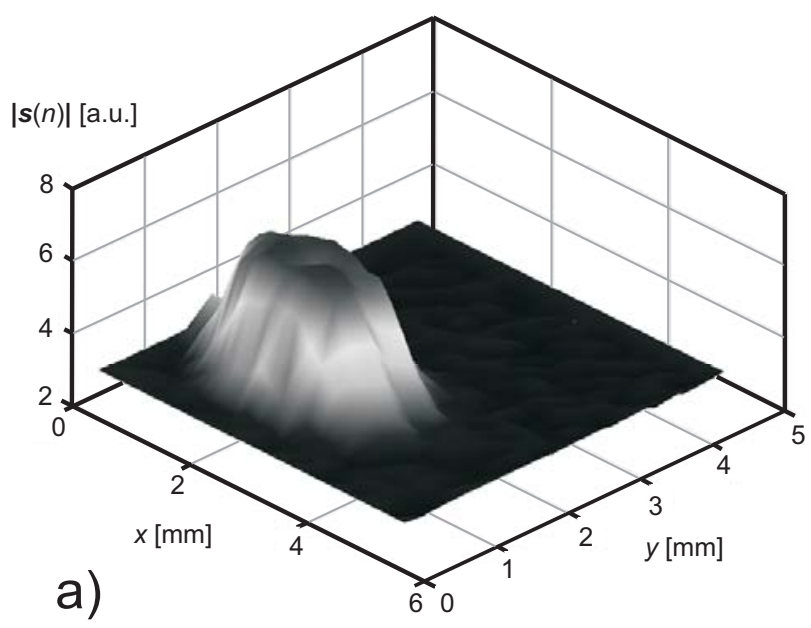

The second sample was made of a block with some drilled holes with angles of $30^{\circ}, 45^{\circ}$ and $90^{\circ}$ in both orthogonal directions. An example of visualization of a drilled hole is shown in Fig. 6. This hole was drilled with an angle of 45 degrees and depth $20 \mathrm{~mm}$. Each drilled hole on the sample was tested as described in section 2.

\section{Conclusions}

A simple novel method for 3D visualization of flaws during eddy current testing based on the Fourier transform was presented in this paper. The described visualization method is simple and uses the well-known FFT (Fast Fourier Transform). FFT computation libraries are available for each currently widely-used family of microcontrollers.

The method is limited to use for flaws rising from plain surfaces of a tested object during inspection by an automated scanning system using motorized eddy current probe positioning. Flaw depth was not assessed.

This method of visualization is simple in principle and is currently targeted for use in EC scanning devices as the first approximation of an EC scan result. Each detected flaw should be diagnosed and classified by further data processing methods, e.g. using pattern recognition.

\section{Acknowledgments}

This study was supported by research program No. MSM 6840770015 "Research of Methods and Systems for Measurement of Physical Quantities and Measured Data Processing" of the CTU in Prague, sponsored by the Ministry of Education, Youth and Sports of the Czech Republic.

\section{References}

[1] Kreidl, M. et al.: Diagnostic Systems. 1. ed. (in Czech). Praha: CTU, 2001. 352 p. ISBN 80-01-02349-4.

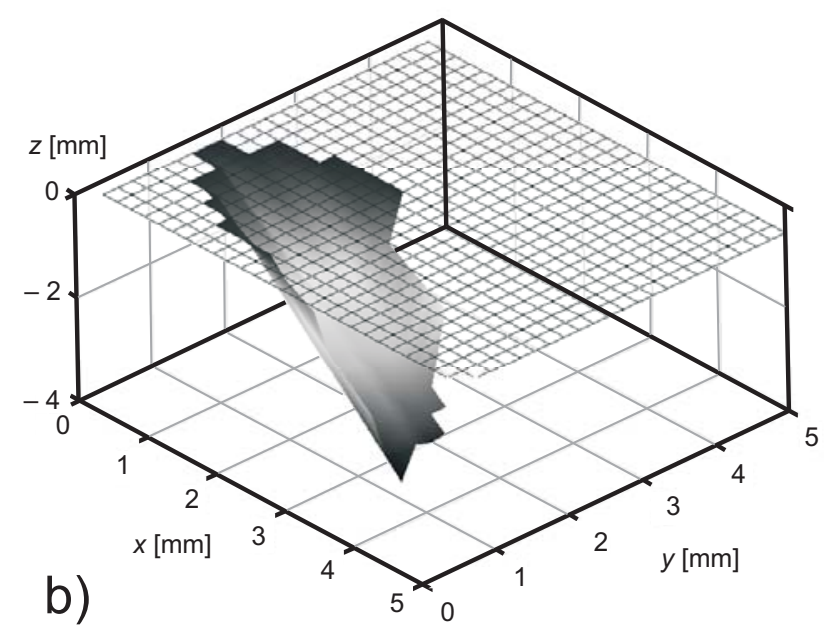

Fig. 6: Visualization of a hole drilled with the angle of 45 degrees on the second sample (a - absolute value of tester response, b - calculated visualization of the flaw) 
[2] Magele, C., Renhart, W., Brandstätter, B.: "Identification of Hidden Ferrous 3D Objects Using Finite Elements." COMPEL: Int J for Computation and Maths. in Electrical and Electronic Eng. Vol. 3 (2001), p. 689-698, Emerald Group Publishing Limited, 2001.

[3] Prémel, D., Pichenot, G., Sollier, T.: "Development of a 3D Electromagnetic Model for Eddy Current Tubing Inspection Current Data." International Journal of Applied Electromagnetics and Mechanics. Vol. 18 (2003), p. 1-5, IOS Press, 2003.

[4] Xiang, P., Ramakrishnan, S., Cai, X., Ramuhalli, P., Polikar, R., Udpa, S. S., Udpa, L.: “Automated Analysis of Rotating Probe Multi-frequency Eddy Current Data From Steam Generator Tubes." International Journal of Applied Electromagnetics and Mechanics, Vol. 12, (2001), p. 151-164.

[5] Šmíd, R., Dočekal, A., Kreidl, M.: “Automated Classification of Eddy Current Signatures during Manual Inspec- tion." NDT \&E International. Vol. 38 (2005), p. 462-470, Elsevier Science, 2005.

Ing. Adam Dočekal

Phone: +420224352346

e-mail:docekaa@fel.cvut.cz

Doc. Ing. Marcel Kreidl, CSc.

Phone: +420224352117

e-mail: kreidl@fel.cvut.cz

Doc. Ing. Radislav Šmíd, Ph.D.

Phone: +420224352131

e-mail:smid@fel.cvut.cz

Department of Measurement

Czech Technical University in Prague

Faculty of Electrical Engineering

Technická 2

16627 Prague 6, Czech Republic 